Research Paper

\title{
Histological Evaluation of Periodontal Ligament in Re- sponse to Orthodontic Mechanical Stress in Mice
}

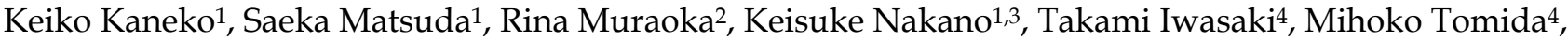 \\ Hidetsugu Tsujigiwa ${ }^{5}$, Hitoshi Nagatsuka ${ }^{3}$, and Toshiyuki Kawakami ${ }^{\circledR}$ \\ 1. Department of Hard Tissue Research, Matsumoto Dental University Graduate School of Oral Medicine, Shiojiri, Japan \\ 2. Department of Orthodontics, Matsumoto Dental University School of Dentistry, Shiojiri, Japan \\ 3. Department of Oral Pathology, Okayama University Graduate School of Medicine, Dentistry and Pharmaceutical Sciences, Okayama, Japan \\ 4. Department of Social Dentistry, Matsumoto Dental University School of Dentistry, Shiojiri, Japan \\ 5. Department of Life Science, Faculty of Science, Okayama University of Science, Okayama, Japan \\ $\bowtie$ Corresponding author: kawakami@po.mdu.ac.jp
}

() 2015 Ivyspring International Publisher. Reproduction is permitted for personal, noncommercial use, provided that the article is in whole, unmodified, and properly cited. See http:/ /ivyspring.com/terms for terms and conditions.

Received: 2015.06.05; Accepted: 2015.07.26; Published: 2015.08.10

\begin{abstract}
The purpose of the study was to determine the cell dynamics in periodontal ligament in response to mechanical stress during orthodontic movement. Following Waldo's method, a square sheet of rubber dam was inserted in between the first and second maxillary molars in $10 \mathrm{ddY}$ mice leaving the stress load for 3 hours. After 3 days and at 1 week, cell count on pressure and tension sides of the periodontal ligament was determined. Furthermore, the type of cell present after mechanical stress was identified using GFP bone marrow transplantation mouse model. Immunohistochemistry was carried out at 0 min (immediately after mechanical stress), 24 hours, 1 week, 2 weeks and 6 months. Temporal changes in the expression of GFP-positive bone marrow derived cells were examined. Moreover, double immunofluorescent staining was performed to determine the type of cell in the periodontal ligament. Cell count on the tension side tremendously increased 3 days after mechanical stress. At 1 week, spindle and round cell count increased compared to the control group. These changes were observed on both tension and pressure sides. Cell count on pressure side at 3 days $(22.11+/-13.98)$ and at 1 week $(33.23+/-11.39)$ was higher compared to the control group $(15.26+/-8.29)$. On the tension side, there was a significantly increased at 3 days (35.46+/-11.85), but decreased at 1 week $(29.23+/-13.89)$ although it is still higher compared to the control group (AD+/-SD: 10.37+/-8.69). Using GFP bone marrow transplantation mouse model, GFP positive cell count increased gradually over time in 6 months. GFP positive cells were also positive to CD31, CD68 and Runx2 suggesting that fibroblasts differentiated into osteoclasts and tissue macrophages. In conclusion, mechanical stress during orthodontic movement promoted the increase in the number of cells in the periodontal ligament on both tension and pressure sides. The increase in the number of cells in the periodontal ligament is believed to be due to the migration and cell division of undifferentiated mesenchymal cells.
\end{abstract}

Key words: Periodontal Ligament, Orthodontic Mechanical Stress, Cell movement, Cell differentiation, Immunohistochemistry

\section{Introduction}

It is well known that orthodontic appliance causes mechanical stress on the periodontal tissues leading to teeth movement. Aside from bone deposition by osteoblasts and bone resorption by osteoclasts, various studies have been done on periodontal tissue remodeling during orthodontic teeth movement.
However, studies on the origin of the fibroblast as well as the cell count in response to orthodontic teeth movement are only very few. Tomida et al. (1) focused on the pluripotency of bone marrow derived cells (BMDCs), since studies have mentioned their migration to the periodontal ligament (PDL) after receiving 
mechanical stress. In addition, Muraoka et al. (2) revealed that BMDCs migrate into the PDL and differentiate into macrophages, osteoclasts, etc.

In the present study, we focused on the histopathological evaluation of cells in periodontal tissue remodeling caused by mechanical stress during orthodontic teeth movement. Furthermore, the source of cells was identified using GFP bone marrow transplantation mouse model by examining GFP-positive cell count immediately after mechanical stress until 6 months. Immunofluorescent double staining was carried out to identify the type of cells, which BMDCs have differentiated.

\section{Materials and Methods}

\section{Laboratory animals}

A total of ten, 8-week old male mice were purchased from SLC Japan Inc. (Hamamatsu, Japan). The mice were housed in metal cage lined with beddings (Paper clean: Peparlet Co., Ltd, Hamamatsu, Japan) in an air-conditioned controlled environment at $24 \pm 1{ }^{\circ} \mathrm{C}$. During breeding, the animals had free access to water and food (Picolab Rodent Diet 20: SLC Japan Inc.).

BMDCs were harvested from ten GFP transgenic mice and were transplanted into 7-week old C57BL/6 mice $(3,4)$. GFP transgenic mice were scarified by ether anesthesia followed by excision of femur, removal of soft tissue and harvest of bone marrow cells. The harvested bone marrow cells were washed with RPMI1640 medium containing antibiotics and then replaced with HBBS. On the other hand, GFP transgenic mice and their 7 week-old cognates were subjected to 10 Gray X-ray irradiation. Seven pieces of $1 \times 10$ squares of bone marrow cells were injected into the tail vein of recipient mice $(5,6)$. After 4 weeks of bone marrow transplantation, histological analysis of various tissues taken from the mouse was carried (Table 1).

Table 1. Periods and Number of Experimental Animals (ddY and GFP)

\begin{tabular}{|c|c|c|c|c|c|c|c|}
\hline \multirow[t]{3}{*}{ ddY } & \multicolumn{6}{|l|}{ Experimental } & \multirow[t]{2}{*}{ Total } \\
\hline & Periods & 3 days & & 1 week & & & \\
\hline & Number & 5 & & 5 & & & 10 \\
\hline \multirow[t]{3}{*}{ GFP } & Experimental & & & & & & \\
\hline & Periods & $0 \min$ & 24 hours & 1 week & 2 weeks & 6 months & \\
\hline & Number & 2 & 2 & 2 & 2 & 2 & 10 \\
\hline
\end{tabular}

\section{Experimental methods}

The animals were subjected to $4.0 \%$ Isofluorane inhalation anesthesia (Isoflu: Animal Science Department, Dainippon Sumitomo Pharma Co., Ltd. Osaka, Japan), which is a mixture of gas and air. The gas anesthesia system (DS Pharma Biomedical Co., Ltd. Laboratory Products, Osaka, Japan) used is for small laboratory animals with adjustable gas concentration and controlled flow rate in order to place the animals under stable condition. After adequate anesthesia was obtained, the mouse was fixed on a handmade bench in a sitting position and the nose attached to a suction hole to maintain general anesthesia. Isofluorane inhalation anesthesia was set to maintain the general anesthesia at a concentration of $1.0 \%$ throughout the experiment. To keep the mouth open during experiment, the maxilla was fixed by tying the upper incisors to the upper part of the bench and the mandible was fixed by applying rubber on the lower incisors to the lower part of the bench. Following Waldo's method, the separator was inserted to induce mechanical stress on the PDL of maxillary molar. The separator used was a rubber dam sheet (Ivory, Premium Rubber Dam Pure Latex: Heraeus Kulzer, GmbH \& Co. KG, Hanau, Germany), cut into approximately $2 \times 2$ square and folded into two. The mouse has 3 maxillary molars on each side numbered as maxillary first molar (M1), second molar (M2), third molar (M3). M1 and M2 have 3 roots and M3 has 2 to 3 roots. The separator was inserted between M1 and M2 and left for 3 hours in order to induce mechanical stress and compression of the PDL. When mechanical load was released, the maxilla with the molar and PL tissue were excised 3 days and 1 week later.

In GFP bone marrow transplanted mice, mechanical load was applied for 3 hours in the same manner as above. After 24 hours, 1 week, 2 weeks and 6 months, the portion of the maxillary periodontal tissue was excised from the maxilla.

For the control or non-treated group, the opposite side of the maxillary molar PDL was used. The distal side of the buccal roots of M1 was used as the observation site. The experiment was carried out following the animal experimental guidelines of the ethics committee of Matsumoto Dental University.

\section{Tissue preparation}

After excision, the tissues were placed in $4 \%$ paraformaldehyde with $0.05 \mathrm{M}$ phosphate buffer fixing solution for 24 hours and then demineralized in 10 $\%$ EDTA solution for 3 weeks. Then after, the specimens were embedded in paraffin; serial sections were made with a thickness of $5 \mathrm{~mm}$ horizontally to the PDL, stained with HE and examined under the light microscope. To confirm the cell count in the PL on the pressure and tension sides, the entire tissue was carefully examined for cell morphology and areas with minimal changes were chosen. Then after, cell count in square pixels using Adobe Photoshop Ele- 
ment 13 was carried out. Note that gaps such as lumen of the blood vessel were excluded.

\section{Immunohistochemistry}

For immunohistochemistry, specimens were deparaffinized in xylene and placed in an incubator at $60{ }^{\circ} \mathrm{C}$ for $30 \mathrm{~min}$. Then after, the specimens were pre-treated in $0.03 \%$ hydrogen peroxide methanol solution and then immersed in $10 \mathrm{mM}$ citric acid buffer with a $\mathrm{pH}$ of 6.0 and placed in autoclave at 121 ${ }^{\circ} \mathrm{C}$ for 5 min.

Anti-GFP ChlP grade ab290 (Abcam, Cambridge, UK) with a dilution of 1:5000 was allowed to react overnight at $4{ }^{\circ} \mathrm{C}$. For the secondary antibody, anti-rabbit polyclonal antibody Simple Stain Mouse $\mathrm{MAXPO}^{\circledR}$ (Nichirei, Tokyo, Japan) was allowed to react, then washed with PBS followed by color development with $\mathrm{DAB}$. In a similar manner, the GFP positive cells were counted under the microscope.

\section{Immunofluorescence Double Staining}

Anti-CD31, anti-CD68 and Runx2 antibodies were combined with anti-GFP rabbit polyclonal anti- body. After deparaffinization, pre-treatment was done with citric acid buffer in microwave for $1 \mathrm{~min}$. Blocking was carried out with $10 \%$ donkey for 30 min, followed by reaction with primary antibody at 4 ${ }^{\circ} \mathrm{C}$ overnight. For secondary antibody, Alexa Fluor R568 Donkey, anti-goat IgG antibodies (Molecular Probes, Japan) and Can Get Signal (Toyobo, Japan) with dilution of 1:200 was incubated for $60 \mathrm{~min}$. Staining of the nucleus with $1 \mathrm{mg} / \mathrm{ml}$ DAPI was carried out for $3 \mathrm{~min}$. Finally, slides were washed with TBS and then mounted using Fluorescent Mounting Medium (DAKO).

\section{Results}

\section{Histopathological findings (ddY mice group: \\ Fig. 1)}

In the control group, the PDL runs radially from the cementum and alveolar bone and the bundles are relatively arranged in order. The spindle shaped fibroblasts have oval nucleus running parallel to the PL fibers.
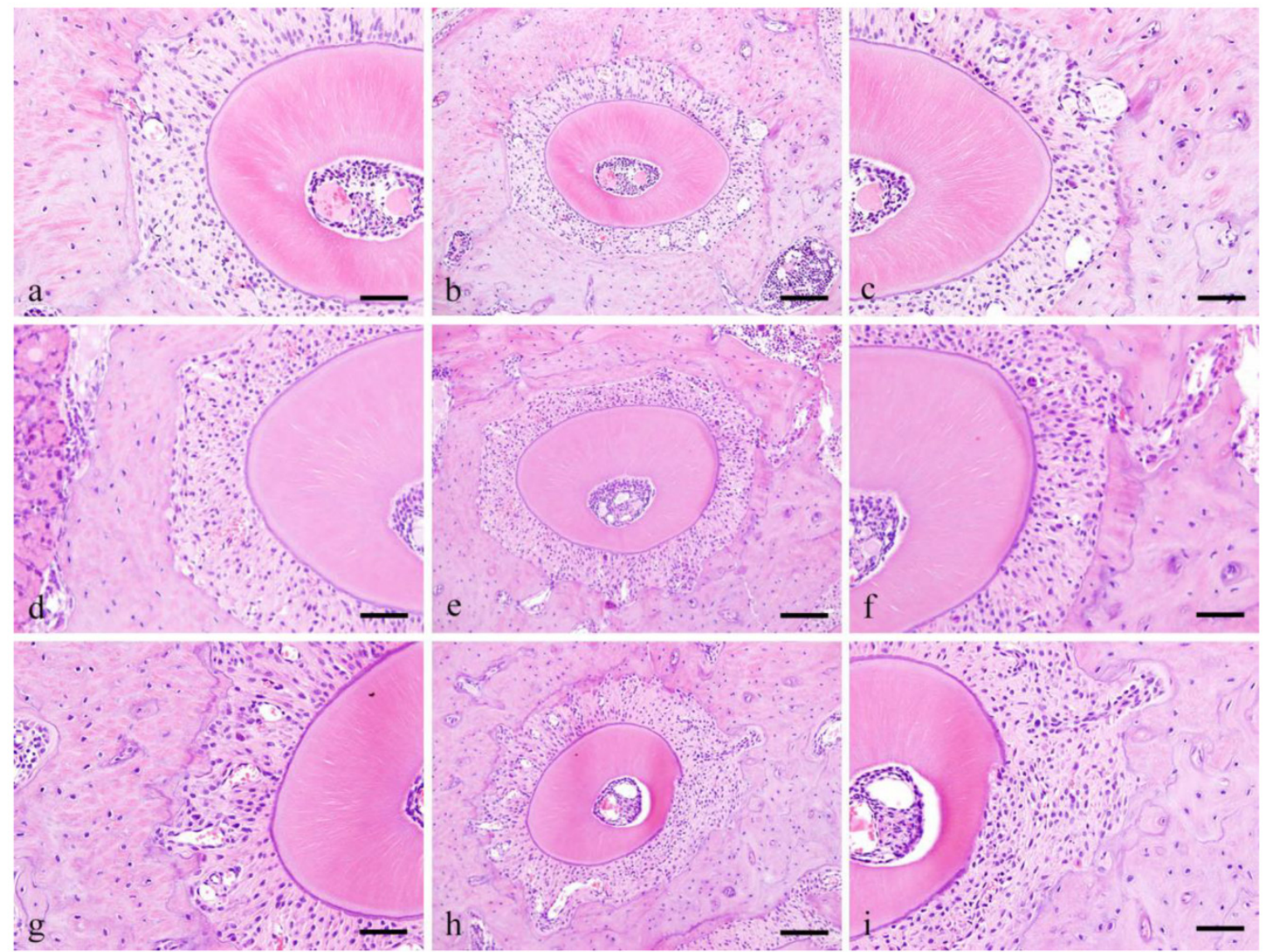

Figure 1. Histopathological views of control specimens. Center column shows whole alveolar bone socket morphology (b, e, f; Scale bar=200 $\mu m$ ). Left

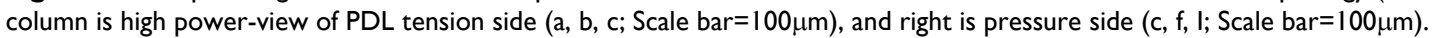


In the Experimental group, at pressure side, 3 days, the PDL space became narrow and densely packed with cells that are irregularly arranged. The size of nucleus varies and the shape is round compared to the spindle shape cells in the control group. Pressure side, 1 week, cell count at 1 week is higher compared to 3 days. Cells were both localized in alveolar bone and cementum and several osteoclasts were observed adjacent to the alveolar bone. The nuclei are huge and occupy most of the cytoplasm and are more round in shape compared to the control group.

Tension side, 3 days, cell count at 3 days is higher compared to the control group. The cells are somehow spindle in shape with round shaped cells in between. Tension side, 1 week, cell count is slight higher compared to 3 days but clearly higher compared to control group. The cells present are both round and spindle in shape.

\section{Cell counting}

Cell count on pressure and tension sides were calculated by counting the number of nuclei in square pixels in selected areas. In pressure side, compared to the control group (15.26 \pm 8.29$)$, a gradual increase in cell count was obtained at 3 days $(22.11 \pm 13.98)$ and continued further at 1 week (33.23 \pm 11.39$)$ (Fig. 2).

In tension side, compared to the control group $(\mathrm{AD} \pm \mathrm{SD}: 10.37 \pm 8.69)$, cell count significantly increased at 3 days $(35.46 \pm 11.85)$ but decreased at 1 week $(29.23 \pm 13.89)$ although it is still higher than the control group. The significant difference was confirmed by Scheffe test (Fig. 3).

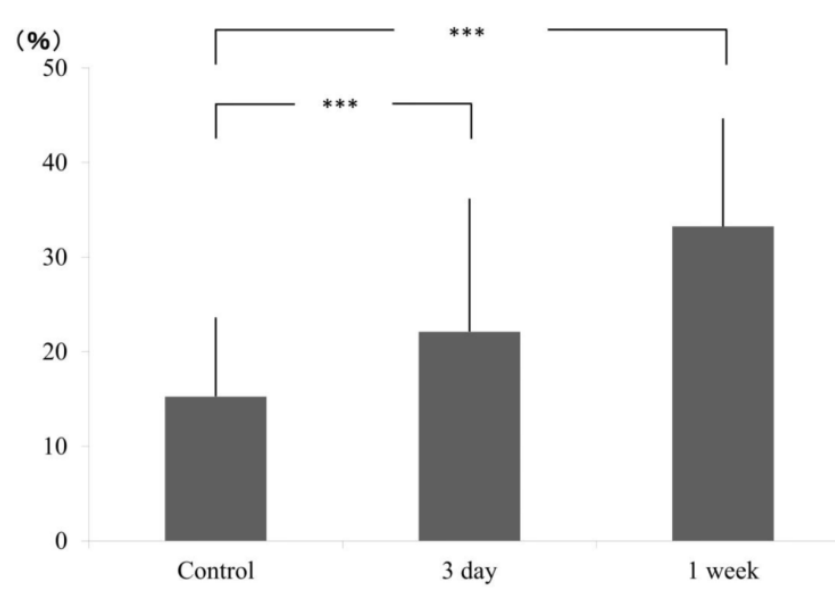

Figure 2. Ratio of pixcel number in the control and experimental (3 day and 1 week), pressure side.

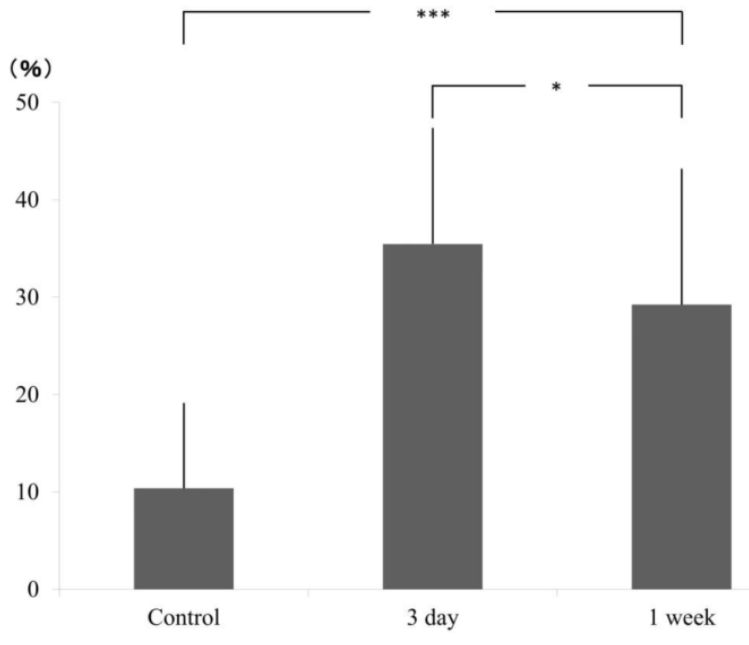

Figure 3. ratio of pixcel number in the control and experimental ( 3 day and 1 week), tension side.

\section{GFP mouse examination}

\section{Histopathological findings}

In the control group, ells on both pressure and tension sides are few. Spindle cells were observed on the pressure side while round cells were mostly observed on the tension side. In 0 min (immediately after mechanical stress loading) specimens, cell count increased on both tension and pressure sides. On the pressure side, the cells are round in shape while on the tension side, the cells are spindle in shape.

In 24 hours, cell count increased on both tension and pressure sides. Spindle shape cells are observed on both sides. In 1 week-specimens, there was no change in cell count on both tension and pressure sides compared to 24 hours. The cells are small and large with round or spindle shape nuclei. In 2 weeks, there was no change in the cell count on both pressure and tension sides but cells are more round in shape on the pressure side. In 6 months specimens, cell count is similar to the control group but round cells are more prominent on the pressure side.

\section{GFP-positive-cell counting}

Immediately after mechanical stress (0 min), some GFP positive cells were observed on the pressure side. At 24 hours, GFP-positive cells slightly increased and the cells are round in shape. At 1 week, GFP-positive cells increased on both pressure and tension sides. At 2 weeks, GFP-positive cells further increased compared to 1 week. At 6 months, GFP-positive cell was predominantly observed on the tension side.

\section{Immunohistochemical findings}

BMDCs are positive to GFP except for those at peripheral capillaries. Most GFP positive cells are 
located in alveolar bone near the PL and exhibit various shapes such as round, spindle, etc. Certainly, GFP-positive cells have the morphology of undifferentiated mesenchymal cells (UMC), which are fusiform, round or even polygonal. In addition, cells lining the lumen of the blood vessel are positive to GFP (Fig. 4a-b).

GFP and immunofluorescent double staining examination deals the specimens were subjected to double staining to identify various cell types of GFP-positive cells. Immunostaining of GFP-CD31, GFP-CD68 and GFP-Runx2 are shown in Fig. 5a-d respectively. Fig. 5 shows GFP positive cells in green fluorescence, Runx 2 positive cells in red fluorescence and superimposition in orange fluorescence. Fusiform cells are arranged in bundles towards the root of the tooth. When superimposed with DAB, the nuclei stained blue and the periphery were stained with orange.

\section{Discussion}

Recently, bone marrow derived un-differentiate mesenchymal cells (UDMC) has been widely used in studies on repair and regeneration of periodontal tissue, bone and other organs. During the regeneration of alveolar bone and PDL, studies have mentioned the differentiation of UDMC from bone marrow into cartilage (7). Experiments on GFP mouse and BMDCs showed that after acinar cell transplantation, the cells differentiated into salivary gland components such as ductal cells and myoepithelial cells with the ability to play a role in salivary gland tissue (8), as well as into olfactory cells (9). Histological experiment on rats subjected to orthodontic load showed that bone marrow stem cells increased the bone remodeling capacity (10).

Tomida et al. (1) measured the increase in PDL cells after mechanical stress at 1 and 5 weeks. In the present study, cell count was determined by counting GFP positive cells in the control and experimental groups. Results showed that GFP positive cell count in the experimental group is significantly higher than the control group. The increase in cell count in the PDL after mechanical stress highly suggests that the transplanted BMDCs were activated to migrate towards the PDL.

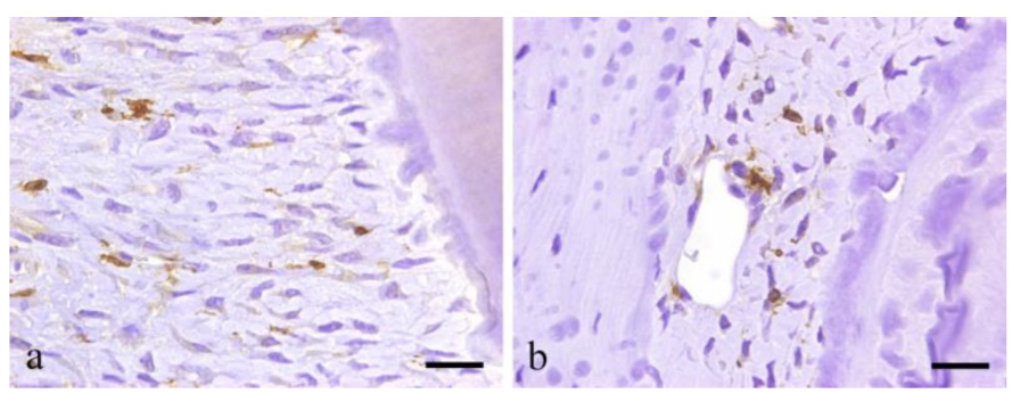

Figure 4. Immunohistochemical results showing GFP-positive round- or spindle-shaped cells within the PDL tissues (a; scale bar=50 $\mu \mathrm{m}$ ), and GFP-positive products existing within the vascular endothelial cells (b; scale bar $=50 \mu \mathrm{m})$.
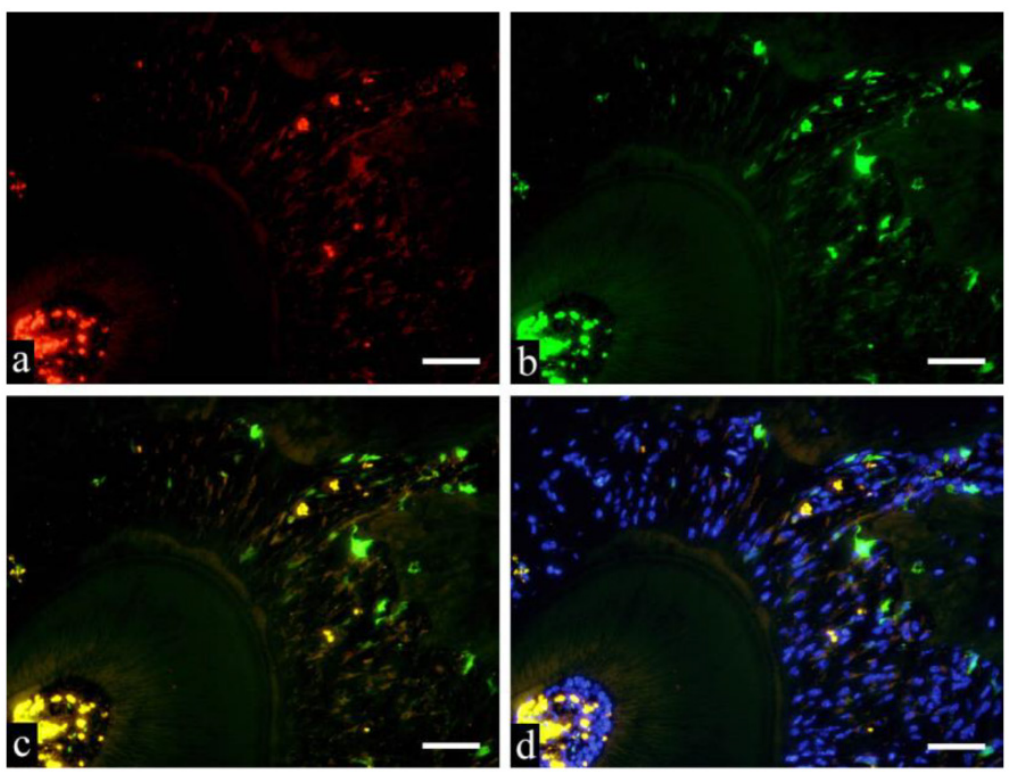

Figure 5. Immunofluorescent staining images of PDL (a: Runx2; b: GFP; c: merged image of Runx2 and GFP; and d: merged image of Runx2, GFP and DAPI; 6 months specimen; scale bar $=100 \mu \mathrm{m})$. 
Tsujigiwa et al. (11), using GFP mouse experiments showed that transplanted BMDCs migrated into the pulp and differentiated into cells that constitute the pulp tissue. Using the same experiment, $\mathrm{Mu}-$ raoka et al. (2) showed that transplanted BMDCs migrated into PDL and then later differentiated into PDL cells. However, the biological reaction of mesenchymal cells in response to mechanical stimulus also led to the change in the cell morphology (12). The migration of transplanted BMDCs using GFP mouse was reported to be due to mechanical stimulation (9).

The results of the study suggested that mechanical stress promoted the increase in cell count in PL on both pressure and tension sides. Cell count increased in as short as 1 week although change in cell morphology was not detected. To determine if the increase in cell count was due to the migration of BMDCs, GFP positive cells were counted in the same manner done by Tomida et al. (1). A gradual increase in GFP positive cell count immediately after stress was noted, which further increased over time until 6 months. It is believed that PL was supplied with BMDCs for a long period of time. Furthermore, immunofluorescent double staining was performed to characterize the cells that have differentiated. Tsujigiwa et al. (13) confirmed that transplanted BMDCs migrate to the area of bone repair shown by double staining with GFP and CD34 in osteoclasts, etc. Applying the methodology of Muraoka et al. (2), we were able to identify the differentiation of cells into osteoclasts and macrophages by double immunofluorescent staining with GFP, CD31, CD68 and Runx2. Results showed that some GFP-positive cells that also expressed CD31 are either BMDCs or vascular endothelial cells and their differentiation was evident. Similar results were obtained with CD68. Moreover, the expression of Runx2 was carried out since it is also expressed by fibroblast (14-16). Results showed that the cells that co-expressed GFP and Runx2 are long and spindle shaped PL fibroblasts strongly suggesting that the cells were derived from bone marrow.

Based on the results and discussion, the following conclusions have been obtained:

1. Orthodontic mechanical stress promotes the growth of cells in the PDL.

2. Using GFP mouse, cell proliferation was brought about by the migration of BMDCs into the PDL.

3. BMDCs that migrated to the PDL differentiated into vascular endothelial cells, macrophages, osteoclasts and fibroblasts.

\section{Acknowledgments}

This study was supported in part by Grant-in-Aid for Scientific Research (C) \#25463204,
(C) \#26463104 and Grant-in-Aid for Young Scientists (B) \#26861804 from the Japan Society for the Promotion of Science.

\section{Competing Interests}

The authors have declared that no competing interest exists.

\section{References}

1. Tomida M, Tsujigiwa H, Nakano K, Muraoka R, Nakamura T, Okafuji N, Nagatsuka $\mathrm{H}$ and Kawakami T. Promotion of transplanted bone marrow-derived cell migration into the periodontal tissues due to orthodontic mechanical stress. Int J Med Sci. 2013; 10: 1321-1326.

2. Muraoka R, Tsujigiwa H, Nakano K, Katase N, Tamamura R, Tomida M, Okafuji N, Nagatsuka $\mathrm{H}$ and Kawakami T. Transplanted bone marrow-derived cell migration into periodontal tissues and cell differentiation. J Hard Tissue Biol. 2011; 20: 301-306.

3. Okabe M, Ikawa M, Kominami K, Nakanishi T, Nishimune Y. 'Green mice' as a source of ubiquitous green cells. FEBS Lett. 1997; 407: 313-319.

4. Ikawa M, Yamada S, Nakanishi T Okabe M. Green flourecsent protein(GFP) as a vital maker in mammals. Curr Top Dev Biol. 1999; 44:1-20.

5. Wall DA, Hamberg SD, Reynolds DS, Burakoff SJ, Abbas AK, Ferrara JL. Immunodeficiency in graft-versus-host disease. I. Mechanism of immune suppression. J Immunol. 1998;140: 2970-2976.

6. Zijlmans JM, Visser JW, Leterveer L, Kleiverda K, Heemskerk DP, Kluin PM, Willemze R Fibbe WE. The early phase of engraftment after murine blood cell transplantation is mediated by hematopoietic stem cells. Proc Natl Acad Sci USA. 1998; 95: 725-729.

7. Cai X, Yang F, Yan X, Yang W, Yu N, Oortgiesen DA, Wang Y, Jansen JA and Walboomers XF. Influence of bone marrow-derived mesenchymal stem cells preimplantation differentiation approach on periodontal regeneration in vivo. J Clin Periodontol. 2015; 42: 380-389.

8. Yuan Y, Tamamura R, Lei L, Katase N, Sathi G, Ito S, Tsujigiwa H and Nagatsuka $\mathrm{H}$. The Ability of transplanted bone marrow-derived cells to differentiate into parenchymal cells of salivary glands. J Hard Tissue Biol. 2013; 22: 433-438

9. Noda Y, Nishizaki K, Yoshinobu J, Orita Y, Tsujigiwa H and Yamada M. The engraftment and differentiation of transplanted bone marrow-derived cells in the olfactory bulb after methimazole administration. Acta Otolaryngol. 2013; 133: 951-956.

10. Ekizer A, Yalvac ME, Uysal T, Sonmez MF, Sahin F. Bone marrow mesenchymal stem cells enhance bone formation in orthodontically expanded maxillae in rats. Angle Orthodontist. 2015; 85(3):394-399.

11. Tsujigiwa H, Katase N, Sathi G, Buery R, Hirata Y, Kubota M, Nakano K, Kawakami $\mathrm{T}$ and Nagatsuka $\mathrm{H}$. Transplanted bone merrow derived cell differentiated to tooth, bone and connective Tissues in mice. J Hard Tissue Biol. 2011; 20: 147-152.

12. Hao J, Zhang Y, Jing D, Shen Y, Tang G, Huang S and Zhao Z. Mechanobiology of mesenchymal stem cells: A new perspective into the mechanically induced MSC fate. Acta Biomater. 2015 Jul;20:1-9.

13. Tsujigiwa H, Hirata $Y$, Katase N, Buery RR, Tamamura R, Ito S, Takagi S, Iida $\mathrm{S}$, Nagatsuka $\mathrm{H}$. The role of bone marrow-derived cells during the bone healing process in the GFP mouse bone marrow transplantation model. Calcif Tissue Int. 2013; 92: 296-306.

14. Watanabe T, Nakano K, Muraoka R, Shimizu T, Okafuji N, Kurihara S, Yamada K and Kawakami T. Role of Msx2 as a promoting factor for Runx2 at the periodontal tension sides elicited by mechanical stress. Eur J Med Res. 2008; 13: 425-431.

15. Yoshizawa $T$, Takizawa F, Izawa F, Ishibashi $O$, Kawashima $H$, Matsuda A, Endo N, Kawashima H. Homebox protein Msx2 acts as a molecular defense mechanism for preventing ossification in ligament fibroblasts. Mol Cell Biol. 2004; 24: 3460-3472.

16. Saito $\mathrm{Y}$, Yoshizawa $\mathrm{T}$, Takizawa $\mathrm{F}$, Ikegami $\mathrm{M}$, Ishibashi $\mathrm{O}$, Okuda $\mathrm{K}$, Ishibashi $\mathrm{K}$, Obinata $\mathrm{M}$ and Kawashima H. A cell line with characteristics of the periodontal ligament fibroblasts is negatively regulated for mineralization and Runx2/Cbfa1/Osf2 activity, part of which can be overcome by bone morphogenetic protein-2. J Cell Sci. 2002; 115: 4191-4200. 